\title{
EL JUEZ CONSTITUCIONAL Y LA REELECCIÓN PRESIDENCIAL EN AMÉRICA LATINA*
}

Ernesto Cárdenas ${ }^{\mathrm{a}}$

Federico Corredor ${ }^{\mathrm{b}}$

*DOI: https://doi.org/10.18601/01245996.v20n38.03. Los autores agradecen los comentarios, sugerencias y aportes del Dr. Mauricio Pérez Salazar; los errores son responsabilidad exclusiva de ellos. Recepción: 14-08-2017, modificación final: 22-01-2018, aceptación: 19-02-2018. Sugerencia de citación: Cárdenas, E. y Corredor, F. (2018). El juez constitucional y la reelección presidencial en América Latina. Revista de Economía Institucional, 20(38), 45-70.

a Doctor en Economía. Profesor asociado, Departamento de Economía, Pontificia Universidad Javeriana, Cali, Colombia, [ernesto.cardenas@javerianacali.edu.co].

b Magíster en Economía. Profesor, Facultad de Economía, Universidad Externado de Colombia, Bogotá, [ivan.corredor@uexternado.edu.co]. 


\section{El juez constitucional y la reelección presidencial en América Latina}

Resumen. Las disposiciones constitucionales sobre reelección presidencial se modificaron en varios países latinoamericanos durante los últimos años. En algunos países esos cambios pusieron en riesgo la existencia de la democracia. Este artículo utiliza el enfoque de principal-agente para analizar el papel del juez constitucional en la reelección presidencial como mecanismo de rendición de cuentas. Encuentra que la reelección inmediata en democracias de fuerte presidencialismo, puede ser un mecanismo perverso que agudiza la concentración del poder y deteriora el bienestar de los ciudadanos en vez de someter a los políticos a rendición de cuentas.

Palabras clave: reelección presidencial, juez constitucional, presidencialismo, modelo principal-agente; JEL: D72, K00

\section{Constitutional judge and presidential reelection in Latin America}

Abstract. Constitutional provisions regarding presidential re-election have been modified in several Latin American countries in recent years. In some countries, these changes put the existence of democracy at risk. This article uses the principal-agent approach to analyze the role of the constitutional judge in presidential re-election as an accountability mechanism. It finds that immediate reelection in democracies of strong presidentialism can be a perverse mechanism that sharpens the concentration of power and deteriorates the welfare of the citizens instead of making politicians accountable.

Keywords: presidential reelection, constitutional judge, presidentialism, principal agent model; JEL: D72, K00

\section{O juiz constitucional e a reeleição presidencial na América Latina}

Resumo. As disposições constitucionais sobre a reeleição presidencial foram modificadas em vários países latino-americanos durante os últimos anos. Em alguns países, essas mudanças colocaram em risco a existência da democracia. Este artigo utiliza o enfoque de principal agente para analisar o papel do juiz constitucional na reeleição presidencial como mecanismo de prestação de contas. Defende que a reeleição imediata em democracias de forte presidencialismo pode ser um mecanismo perverso que aumenta a concentração de poder e deteriora o bem-estar dos cidadãos ao invés de submeter os políticos à prestação de contas.

Palavras-chaves: reeleição presidencial, juiz constitucional, presidencialismo, modelo principal-agente; JEL: D72, K00 
$\mathrm{E}$ 1 objetivo de este artículo es establecer si la reelección presidencial inmediata, acompañada de la presencia de un juez de última instancia como supervisor del acuerdo constitucional, fortalece el proceso político de redición de cuentas en las democracias de América Latina.

Nuestra hipótesis es que, en democracias caracterizadas por un fuerte presidencialismo, como las latinoamericanas, la reelección presidencial inmediata no funciona como mecanismo de rendición de cuentas. Lo anterior como consecuencia de la independencia de la que bajo ciertas condiciones puede llegar a carecer el juez constitucional.

Este artículo se propone evaluar la hipótesis planteada a partir de un análisis histórico-coyuntural de la reelección presidencial y de los pronunciamientos aprobatorios del juez constitucional en diferentes países de América Latina. La línea argumental identifica tres elementos esenciales: el primero es la perspectiva de la rendición de cuentas como un problema de agencia, que se deriva de los problemas de información a los que se enfrenta la ciudadanía al delegar funciones de supervisión en el juez constitucional. El segundo es el potencial de la reelección presidencial inmediata para proveer incentivos al presidente. Finalmente, el tercero es el establecimiento de un juez constitucional para proteger los intereses de los ciudadanos, es decir, que se considera benevolente, y las implicaciones de sus acciones cuando su perfil ético se modifica.

El aporte central de este trabajo consiste en establecer de manera precisa el papel que cumple el juez constitucional en el fenómeno reeleccionista latinoamericano a la luz de un modelo principal-agente con supervisor. Como se verá, sobre el presidencialismo y el proceso reeleccionista en América Latina se ha escrito ampliamente; de igual modo, sobre el activismo judicial y la judicialización de la política hay una extensa literatura; e incluso, sobre la relación entre la reelección presidencial y el juez constitucional hay también una producción académica considerable; sin embargo, no se ha estudiado la interacción de estos fenómenos desde la perspectiva teórica de la economía de la información, y más puntualmente desde los problemas de agencia, que brindan elementos claros de análisis para la toma de decisiones de política.

Este trabajo contiene cuatro secciones incluyendo esta introducción. La segunda sección establece los elementos teóricos que permiten entender el proceso de rendición de cuentas desde la perspectiva de la teoría de agencia; en ella se discute el papel del juez constitucional y su relación con la reelección presidencial. En la tercera sección se expone el papel que históricamente ha tenido la reelección presidencial 
en el continente, así como la acción del juez constitucional en su rol de supervisor. La última sección presenta los principales resultados del trabajo.

\section{EL PROCESO POLÍTICO DE RENDICIÓN DE CUENTAS COMO UN PROBLEMA PRINCIPAL-AGENTE}

El propósito de este artículo es determinar si la reelección presidencial y el juez constitucional, de manera conjunta, fortalecen la rendición de cuentas en los países de América Latina. En la medida que el proceso electoral implica delegación de funciones entre la ciudadanía y sus representantes, relación determinada por la presencia de problemas de información, la perspectiva teórica del modelo principal-agente resulta pertinente para abordar el problema planteado (Laffont, 2000; Laffont y Martirmort, 2002; Besley, 2006; Brandt y Svendsen, 2013). Como lo exponen, entre otros, Pérez (2007) y Gailmard (2012), esta teoría es un marco natural para estudiar la rendición de cuentas en las instituciones políticas, toda vez que provee un marco teórico flexible para modelar innumerables variaciones en los arreglos institucionales y comparar su potencial para inducir resultados deseables en el comportamiento de los agentes.

El problema de la rendición de cuentas debe ser entendido a nivel constitucional, debido a que es allí donde se establece el conjunto de normas y regulaciones bajo las cuales los individuos interactúan y se relacionan (Elster, 1994; Cooter, 2000; Pérez, 2007). La constitución, como función de bienestar social que se establece por medio de un mecanismo político, estipula como objetivo de la comunidad la provisión de bienes públicos y la vigilancia en su producción (Laffont, 2000; Drazen, 2000; Persson y Tabellini, 2000; Drazen, Persson, Tabellini y Saint-Paul, 2000).

La gráfica 1 ilustra las características esenciales del modelo principal-agente propuesto. En la constitución los ciudadanos (que son el principal) tienen un acuerdo explícito con respecto al bienestar derivado del balance entre las transferencias monetarias hechas al gobierno, en cabeza del presidente (quien es el agente), para la provisión de bienes públicos y el nivel óptimo de gasto que este ejecuta, además, frente a la delegación de la supervisión del acuerdo constitucional en el juez constitucional (quien es el supervisor). La relación de agencia se configura cuando los ciudadanos eligen un presidente para que gobierne en su favor. El problema de información radica en que es el agente quien tiene las capacidades específicas para gobernar, los ciudadanos no, y que en el cumplimiento de sus funciones el presidente maneja 
información privilegiada para la toma de decisiones que es desconocida por los ciudadanos. Es decir, existe un problema de información asimétrica a favor del agente. Los ciudadanos, al no estar en capacidad de controlar sus acciones por los problemas de información que enfrentan, delegan al juez para supervisar la labor del presidente y así garantizar a la ciudadanía el cumplimiento del acuerdo fundamental entre ellos y el presidente. Sin embrago, el juez y el presidente podrían coludir en detrimento de la ciudadanía y, por ejemplo, por medio de la reelección del presidente obtener mayores beneficios para ambos. De allí la importancia del proceso político de rendición de cuentas, pues se asume que este mejora el bienestar de los ciudadanos en tanto premia o castiga el desempeño de los servidores públicos (Przeworski, Stokes y Manin, 1999; Maskin y Tirole, 2004; Samuels y Hellwig, 2010).

\section{Gráfica 1}

Relación de agencia en el proceso de rendición de cuentas

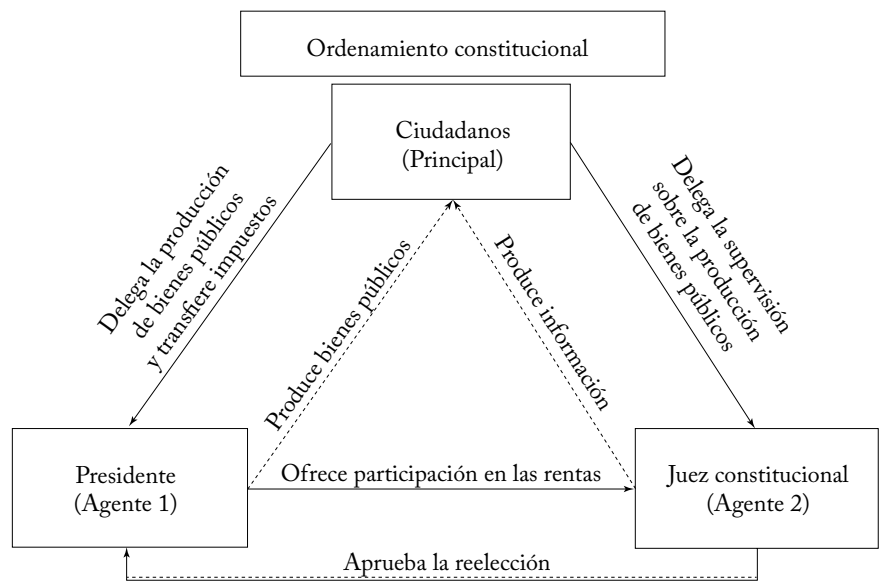

Fuente: elaboración propia, a partir de Laffont (2000).

En principio, la relación entre el juez y el presidente, como materialización del principio de separación de poderes ${ }^{1}$, favorece la rendición de cuentas solo bajo un esquema apropiado de pesos y contrapesos que supone la existencia de un conflicto de intereses entre las distintas ramas del poder público y, a su vez, acuerdo y coordinación en la toma

${ }^{1}$ La rendición de cuentas suele ser entendida, o bien como elecciones periódicas, o bien como la vigilancia y el equilibrio de la separación de poderes. La rendición de cuentas horizontal es vista entonces como la supervisión de una agencia del Estado sobre otra, donde la primera tiene capacidad real para tomar acciones cuando la última actúa contra la ley (O’Donnell, 2003; Kenney, 2003). 
de decisiones de política pública. Esta división de poderes se supone fomenta la competencia entre agentes y disminuye los riesgos de la colusión y la captura del regulador (Laffont y Meleu, 2001; Laffont y Martimort, 1998; Laffont y Martimort, 1999). Ahora bien, la gráfica anterior solo funciona cuando las reglas constitucionales se respetan, la rama judicial es independiente y benévola, y además tiene la capacidad de hacer cumplir las reglas (Persson, Roland y Tabellini, 1997). Sin embargo, la razón subyacente al presente trabajo es precisamente que no hay un esquema equilibrado de pesos y contrapesos dado el poder que concentra el presidente, poder que le permite, entre otros, cambiar reglas constitucionales establecidas como las referentes a la duración de los periodos electorales y la reelección presidencial.

La ausencia de la rama legislativa en el análisis propuesto es la principal expresión de la concentración de poder en la figura del presidente, elemento característico del entorno institucional latinoamericano, y que conduce a escenarios propicios para el abuso del poder (Gargarella y Courtis, 2009; Mainwaring y Pérez-Liñán, 2013). E1 monopolio de la iniciativa legislativa que ostenta el ejecutivo es la razón fundamental para excluir del análisis a la rama legislativa. En los regímenes presidencialistas el ejecutivo propone la agenda del congreso (Carey y Shugart, 1998; Cox y Morgenstern, 2001), y estratégicamente logra mayorías o coopta miembros del congreso para garantizar la aprobación de sus proyectos, lo que implica que la frontera entre las funciones de las ramas del poder público es débil y difusa, los partidos políticos no se estructuran sobre plataformas ideológicas sólidas y el poder legislativo no cumple con su labor de control (Zielinski, Slomczynski y Shabad, 2005). Así, la rama judicial toma el papel protagónico al suplir, en teoría, los vacíos de poder y la ausencia de control producto de la inoperancia del legislador (Landau, 2010; Henao, 2013).

En consecuencia, el ordenamiento constitucional que se propone en este artículo contempla dos mecanismos que procuran proveer los incentivos adecuados para que el presidente actúe en concordancia con el bienestar general; estos son: la reelección presidencial inmediata y la presencia de un juez de última instancia como salvaguardia del acuerdo constitucional.

\section{LA REELECCIÓN PRESIDENCIAL COMO MECANISMO DE RENDICIÓN DE CUENTAS}

Con base en la percepción colectiva de que los políticos son conscientes de las necesidades de su país y están calificados para guiar la ejecución de políticas públicas (Madison, 1787; Maskin y Tirole, 
2004), los ciudadanos votan por un presidente en las elecciones democráticas. Una vez elegido, el presidente tiene acceso a los recursos públicos para implementar políticas que mejoren las condiciones de vida de los ciudadanos. Después, en una nueva elección democrática, dependiendo de su rendimiento (Barro, 1973; Ferejohn, 1986), los ciudadanos deciden si permitir al mandatario mantenerse en el poder por un nuevo periodo o elegir otro presidente. En ausencia de supervisión hay mayor espacio para la extracción de rentas particulares por parte del presidente en detrimento del bienestar del resto de la población (Laffont y Tirole, 1990 y 1993; Acemoglu et al., 2005). El problema básico y que demanda mayor atención es el diseño de un mecanismo que controle y obligue a los políticos a cumplir.

La reelección soluciona los problemas de información asimétrica propios del sistema electoral (Berganza, 2000; Maskin y Tirole, 2004). Por un lado, corrige el riesgo moral mediante la provisión de incentivos para orientar las acciones del agente en dirección de los objetivos del principal; por el otro, tiene un efecto correctivo de la selección adversa en la medida que el electorado puede prescindir de aquellos políticos que tienen intereses opuestos a los suyos. Es decir, la reelección presidencial es vista como un mecanismo para que la ciudadanía se beneficie de la continuidad de un gobierno que considera apropiado, de modo que favorece la rendición de cuentas en tanto alinea los incentivos del presidente con los de los votantes. Por otro lado, gobiernos extensos también pueden tener ventajas en la ejecución de políticas públicas dado que las otras ramas del poder público se muestran renuentes a apoyar gobiernos de corta duración (Carey, 2003).

Sin embargo, como muestra la gráfica 2 , la utilidad de los periodos electorales fijos está determinada por el comportamiento oportunista de los gobernantes. Por comportamiento oportunista en este trabajo entendemos aquel en el cual el presidente prefiere satisfacer sus intereses particulares en detrimento de los de la ciudadanía. Si el oportunismo es alto, un solo periodo es mejor desde el punto de vista del bienestar pues los políticos estarían dispuestos a ir a extremos para ser reelegidos (Streb, 1999; 2005). La imposición de periodos electorales fijos elimina los beneficios que recibe el gobernante por mantenerse varios periodos en el cargo y lo induce a implementar políticas que están más cerca de sus preferencias privadas (Smart y Sturm, 2013; Besley y Case, 1995; Ferraz y Finan, 2011). Si el grado de oportunismo disminuye, las políticas elegidas en el pasado se convierten en el indicador de las verdaderas preferencias del gobernante, y en la señal con 
la cual los votantes pueden detectar y remover políticos equivocados. Así, la combinación de un número limitado de reelecciones posibles, seguido de la certeza del fin del periodo, parece ser una alternativa prominente (Ferejohn, 1986).

Gráfica 2

Perfil del presidente según el grado de oportunismo

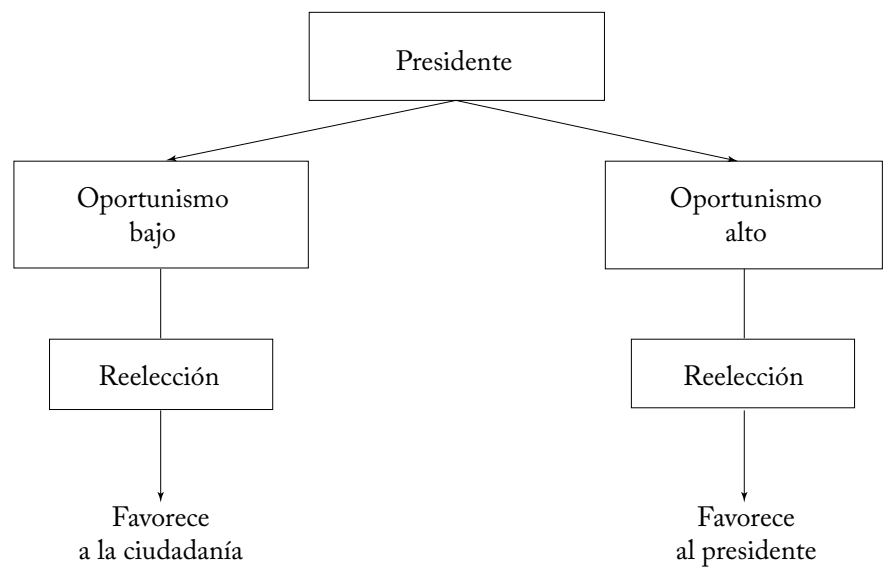

Fuente: elaboración propia.

Sin embargo, ante la posibilidad de reelección, el valor de mantenerse en el poder depende de la dimensión de las rentas acumuladas; es decir, la riqueza y el estatus obtenidos después de haber ejercido el poder. La extracción de rentas y la propensión a subvertir las instituciones por parte de los presidentes depende en gran medida de su capacidad para cooptar legislaturas y asegurar cambios constitucionales (Baturo, 2014). En años recientes, muchos presidentes en el mundo han decidido mantenerse en el poder una vez su periodo inicial máximo se ha vencido, modificando así la constitución, y algunas veces reemplazándola en su totalidad (Ginsburg, Melton y Elkins, 2010).

Por consiguiente, en presencia de un electorado desinformado, un elevado costo de acceso a la información y una retroalimentación lenta de las implicaciones políticas del rendimiento de los mandatarios, las decisiones tomadas por burócratas (que no son sujeto de elección democrática) pueden ser una alternativa más prometedora (Maskin y Tirole, 2004). La posibilidad de que el político electo se congracie con la opinión pública mediante beneficios de corto plazo, pero sin ningún programa estructural que incremente el bienestar de los ciudadanos en el largo plazo, conduce al análisis del papel del juez, y al balance entre dejar las decisiones de política pública a un político elegido o a 
un burócrata cuyo cargo no es de elección popular y que en teoría no está sujeto a los vaivenes de la opinión pública y la dinámica electoral.

\section{EL JUEZ DE ÚLTIMA INSTANCIA COMO SUPERVISOR DEL ACUERDO CONSTITUCIONAL}

El argumento central de este trabajo sostiene que la reelección presidencial como mecanismo de rendición de cuentas no puede entenderse en su totalidad sin tener en cuenta el papel que cumple el juez constitucional.

Dado que la constitución estipula los periodos electorales y las restricciones a la tenencia del poder, el pronunciamiento del juez como salvaguardia del acuerdo constitucional sobre la reelección presidencial es condición necesaria para la existencia de esta figura jurídica. El juez constitucional condicionará el interés del presidente en ser reelegido, toda vez que la fortaleza e independencia judicial son factores que favorecen o frustran significativamente los intentos del ejecutivo por permanecer en el poder (Magaloni, 2003; Baturo, 2014). Como consecuencia, la agenda del juez involucra un debate sobre los intereses políticos coyunturales y las transformaciones sociales de largo plazo (Schor, 2009). Así, el juez, o bien favorece la extracción de rentas en el escenario de colusión (Tirole, 1986; Strausz, 1997; Baliga, 1999; Faure-Grimaud et al., 2002), o bien se establece como actor de las políticas públicas: su obligación de salvaguardar los mandatos constitucionales lo conduce a identificar situaciones socialmente relevantes, formular soluciones y demandar su cumplimiento (Henao, 2013).

Desde esta perspectiva, la efectividad de este mecanismo depende en gran medida de la condición ética del juez. En consecuencia, el modelo planteado establece que el juez puede ser de dos tipos: un juez maximizador de rentas o un juez benevolente. El juez maximizador de rentas es un agente dispuesto a aceptar o a exigir parte de las rentas extraídas por el presidente para que su pronunciamiento favorezca la reelección presidencial. El escenario del juez maximizador de rentas es aquel en el cual el presidente logra consolidar mayorías al interior de las cortes supremas. Es decir, la acción de los jueces hace parte de la estrategia política del presidente y no garantiza la estabilidad ni el cumplimiento del acuerdo constitucional. Por su parte, el juez benevolente es un agente cuyo pensamiento y acción están definidos por un conjunto de ideas fundamentales ligadas a una lectura de la constitución. Es aquel que mediante sus pronunciamientos representa los intereses de la ciudadanía 
al interpretar en su favor las disposiciones constitucionales ${ }^{2}$, y sin tener en cuenta los intereses particulares del presidente. Este juez aprueba o rechaza la reelección presidencial por consideraciones sobre el bienestar de los ciudadanos, y no sobre los beneficios del presidente, como el juez maximizador de rentas. La gráfica 3 sintetiza el argumento.

Esta diferenciación de los perfiles éticos del juez busca dirigir la discusión hacia la creación de mecanismos que procuren cierres de segunda instancia, pues la presencia de un juez maximizador de rentas conduce a la compleja discusión sobre quién supervisa al supervisor (Hurwicz, 2008; González 2008), y también a la noción de captura del regulador (Stigler, 1971; Tirole, 1986; Laffont y Martimort, 1991; Dal Bó, 2006). Un cierre de primera instancia no es posible en la medida que la rama legislativa no cumple su labor de control, de manera que la presencia del juez constitucional, en caso de ser uno de tipo benevolente, resulta ser un cierre de segunda instancia, subóptimo, pero bastante aceptable (González, 2008). Es decir, en el lenguaje de Hurwicz, el juez maximizador de rentas representa al tipo de guardián que tiene que ser vigilado, mientras que el código ético del juez benevolente lo hace equiparable con el guardián platónico que cumple su labor a cabalidad.

Gráfica 3

Perfil del juez según su inclinación ética

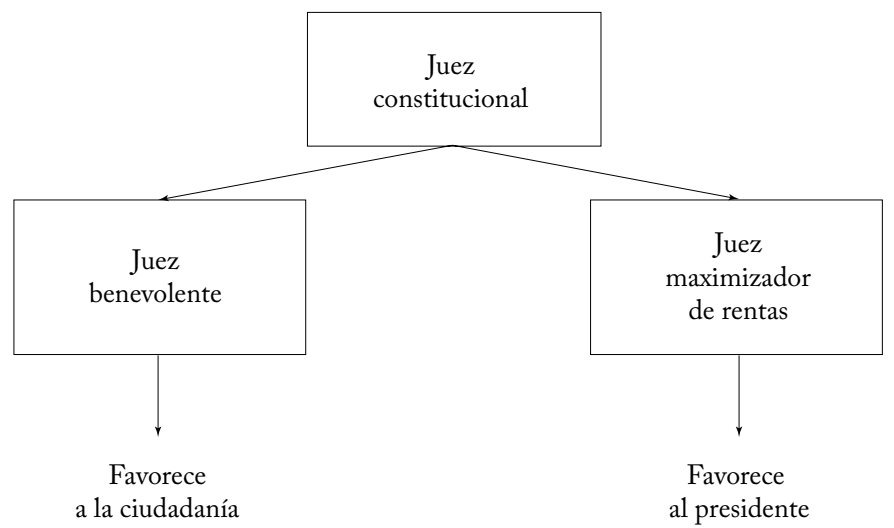

Fuente: elaboración propia.

${ }^{2}$ Como ejemplo, conforme al Decreto 2067 de 1991, la Corte Constitucional colombiana se pronuncia "en nombre del pueblo y de la constitución". Y como aproximación al poder de la Corte en su función interpretativa, la sentencia C-113 de 1993 dice: "entre la Constitución y la Corte Constitucional, cuando esta interpreta aquella, no puede interponerse ni una hoja de papel”. 
E1 punto relevante es determinar si, en la defensa de la constitución, la interpretación que el juez hace de la información favorece a la ciudadanía o al presidente. El juez constitucional, se asume, procesa información relevante que la ciudadanía no está en capacidad de comprender y supervisa así las acciones del presidente mediante la defensa del acuerdo constitucional. La doctrina de la sustitución de la constitución materializa con precisión esta problemática, pues dicta que la constitución tiene unos elementos esenciales que definen su identidad, y el juez constitucional decide si una reforma entra en contradicción con estos elementos (Bernal, 2013; Roznai, 2017). Pero aunque se ha establecido en forma precisa cómo evaluar la sustitución, los criterios y procedimientos ${ }^{3}$, y pese a que existe un instructivo claro, el juicio de sustitución no puede ser totalmente objetivo. El juez en su función interpretativa puede, eventualmente, considerar pertinente la reelección presidencial, argumentar en su favor y, en consecuencia, aprobarla; sin embargo, esta interpretación no carece de sesgos ideológicos, y está basada en concepciones políticas preconcebidas (Shapiro y Sweet, 2002; Nunes, 2009). En este sentido, la aprobación de la reelección presidencial por parte del juez, si bien puede estar guiada por un objetivo loable de fidelidad al acuerdo constitucional, puede asimismo representar un apoyo al gobernante de turno.

Algunos autores (Martínez-Barahona, 2010; Martínez-Barahona y Brenes, 2012) han propuesto que la rama judicial será la que apruebe la reelección presidencial cuando el presidente parezca tener suficiente apoyo popular, controle una corte proactiva y falten mayorías en la rama legislativa. Su conclusión apunta hacia los riesgos derivados de judicializar la política con el traslado de estas decisiones a las cortes supremas (Uprimny, 2007; Kapiszewski y Taylor, 2008; MartínezBarahona, 2009). En consecuencia, es fundamental establecer los límites del poder que concentra el juez; es decir, el necesario para actuar con eficacia y el suficiente para no extralimitarse en sus funciones. El equilibrio requerido para que este agente ejerza la función de supervisión y asimismo sea sujeto de rendición de cuentas es el tema central a la hora de evaluar la pertinencia de las acciones de esta rama del poder público.

\footnotetext{
${ }^{3}$ Para un análisis exhaustivo puede consultarse la jurisprudencia de la Corte Constitucional colombiana al respecto en la sentencia C-551 de 2003, así como las sentencias C-970 y C-971 de 2004 y C-1040 de 2005, en las cuales se delineó el método para realizar el examen de sustitución.
} 


\section{MODELO DE CONTROL DE LOS POLÍTICOS CON EL JUEZ CONSTITUCIONAL COMO SUPERVISOR}

En esta sección se exponen los principales resultados del modelo propuesto para entender la relación principal-agente en el proceso político de rendición de cuentas en América Latina, como fue planteado en la gráfica 1. El modelo que proponemos es una extensión del planteado por Barro (1973) y su versión matemática se presenta en el anexo que figura en la página web de la revista.

La eficacia de la reelección presidencial inmediata como mecanismo de rendición de cuentas depende fundamentalmente de dos elementos: de la valoración que el presidente haga de sus posibles rentas futuras, dado su nivel de oportunismo o de impaciencia, y de la independencia del juez constitucional frente al presidente, según la naturaleza ética o tipo de juez. En ausencia de un supervisor, y en presencia de información asimétrica, el presidente logrará extraer la mayor cantidad de rentas al imponer el nivel de bienes públicos que maximiza sus preferencias.

Si el presidente valora mucho el presente (el factor de descuento es muy alto), la mejor alternativa para que la información verdadera se revele es establecer un solo periodo de mandato. E1 factor de descuento subjetivo establece la impaciencia del presidente, qué tanto valora el presente o el futuro. Si el grado de impaciencia es bajo, la reelección presidencial podría ser efectiva, pues para maximizar su beneficio el presidente actuará conforme al interés general, al menos en el primer periodo de mandato.

El papel del juez constitucional se considera como un ancla que ata el funcionamiento del Estado a unos principios generales establecidos por la comunidad política. No obstante, entornos institucionales caracterizados por la preponderancia que tiene la rama ejecutiva frente a las demás ramas del poder público (fuerte presidencialismo) se alejan cada vez más de su naturaleza democrática en la medida que el juez pierde independencia frente al ejecutivo. En este escenario, los pronunciamientos del juez tenderán a ser cada vez más cercanos a los intereses del presidente, y no a los de los ciudadanos; o lo que es lo mismo, al acuerdo constitucional.

El modelo propone que el presidente y el juez maximizador de rentas desean una mayor provisión de bienes públicos que los electores. Sin embargo, la evidencia sugiere que suele haber poca provisión de bienes públicos dados los impuestos que pagan los contribuyentes. La brecha entre el mayor gasto y la menor provisión de bienes públicos obedece, al menos en parte, a la extracción de rentas de unos y otros. Es 
decir, los ciudadanos enfrentan una disyuntiva frente a la provisión de bienes públicos; derivan satisfacción de su uso, pero tienen que pagar por ellos. Para el político y el juez, los bienes públicos representan la oportunidad de extraer un mayor nivel de rentas.

Por tanto, en presencia de un juez maximizador de rentas, y dada la capacidad del ejecutivo de capturar la rama judicial a través de transferencias monetarias, los ciudadanos no deben contemplar la reelección presidencial inmediata como mecanismo de rendición de cuentas en su ordenamiento constitucional.

Ahora bien, la figura del juez benevolente, que no está supeditada a exigir ninguna transferencia al agente para alterar la información que dará al principal frente al nivel óptimo de gasto, permitiría alinear los intereses de los ciudadanos con los del presidente, al menos durante el primer periodo de mandato. De manera que la figura del juez constitucional resultaría ser una alternativa plausible de supervisión si lograra estructurarse un esquema de incentivos que garantice la existencia de un juez benevolente, y que disminuya en lo posible los elementos del entorno institucional que estimulen el surgimiento de un juez maximizador de rentas.

En consecuencia, un ordenamiento constitucional que procure una implementación exitosa de mecanismos de rendición de cuentas debe sopesar los eventuales beneficios a corto plazo de la reelección presidencial y los costos para la estabilidad del Estado y sus principios democráticos. Asimismo, el papel del juez constitucional debe ser analizado en detalle. Las condiciones institucionales en que un presidente concentra mucho poder y los personalismos determinan el éxito en la contienda electoral, de modo que la sustitución de la constitución es relativamente frecuente (características propias de regímenes en tránsito hacia el autoritarismo) y el papel del juez como supervisor del acuerdo constitucional es determinante. No obstante, sus acciones contienen una amenaza intrínseca, $\mathrm{y}$ si son consecuencia de un activismo que no reconoce límites el principio medular de la separación de poderes se vería aún más perjudicado.

\section{LA EXPERIENCIA LATINOAMERICANA}

\section{El fuerte presidencialismo como expresión del} alto grado de oportunismo del presidente

E1 marco teórico y los resultados del modelo resaltan dos elementos esenciales para entender la rendición de cuentas en América Latina: el grado de oportunismo o impaciencia del presidente y la acción del 
juez constitucional según su perfil ético. El problema de agencia radica en la dificultad de la ciudadanía para identificar las inclinaciones reales de los dos agentes. En este orden de ideas, el análisis histórico de la reelección y del papel del juez constitucional indica que el grado de oportunismo del presidente es muy alto en América Latina, y que el empoderamiento del juez puede favorecer la concentración del poder y la extracción de rentas. Es decir, que el mecanismo conjunto de reelección y acción del juez va en detrimento del bienestar de la población.

Desde finales de los años 70 y hasta principios de los años 90, la mayoría de los países en América Latina experimentaron la transición del autoritarismo a la democracia, como lo muestra el cuadro 1. De acuerdo con Mainwaring y Pérez-Liñán (2013), en 1977 solo tres países eran regímenes semidemocráticos, mientras que en 1995 diecinueve estaban en esta categoría ${ }^{4}$. Sin embargo, rupturas constantes en el proceso de consolidación de la democracia los llevan a concluir que desde finales de los años noventa hay un retroceso evidente. Estancamiento y erosión de la democracia es como los autores definen esta tendencia. Este retroceso ha sido determinado principalmente porque algunos países que pasaron de ser autoritarios a semidemocráticos no llegaron a consolidar del todo los procesos democráticos, a pesar del tiempo que ha transcurrido desde su primer avance. Asimismo, en algunos casos las características democráticas están en riesgo. Desde la perspectiva de este trabajo, la reelección presidencial y un régimen de fuerte presidencialismo constituyen una combinación indeseable que pone en riesgo la estabilidad de las instituciones y favorece la transición hacia un régimen no democrático (Carey, 2003; Serrafero, 2011; Bernal, Caicedo y Serrafero, 2015).

Los cambios constitucionales que han ocurrido entre 1978 y 2014 tienen dos explicaciones. Por un lado, el rechazo a la larga permanencia en el poder, dada la experiencia de las dictaduras y del autoritarismo ${ }^{5}$;

${ }^{4}$ En el entendido de que los regímenes políticos pueden ser clasificados como competitivos o autoritarios, la ola democrática es vista como el incremento notable y permanente de la proporción de regímenes competitivos. Estos son aquellos que no transgreden de manera flagrante ninguno de los principios de sufragio universal, garantía efectiva de derechos políticos y civiles, y poder político real del gobierno elegido (Mainwaring y Pérez-L., 2013).

${ }^{5}$ Entre estos: México, donde Porfirio Díaz gobernó desde 1876 hasta 1919; República Dominicana, gobernada por Rafael Leonidas Trujillo desde 1930 hasta 1961; Honduras, en donde Tiburcio Carías Andino fue dictador desde 1933 hasta 1949; Nicaragua, con la dictadura de la familia Somoza desde 1935 hasta 1979, y Paraguay, con Alfredo Stroessner desde 1954 hasta 1989. En otros, gobiernos militares o de facto mantuvieron el poder mientras respetaban las características del orden constitucional, incluyendo elecciones y disposiciones frente 
Cuadro 1

Tendencia de los regímenes políticos en América Latina

\begin{tabular}{|c|c|c|c|c|c|}
\hline País & Autoritario & & Democrático & Semi-democrático & Categoría actual \\
\hline Argentina & $1976-1982$ & & $1983-2010$ & & Democrático \\
\hline Bolivia & $1964-1981$ & & $1982-2006$ & $2007-2010$ & $\begin{array}{l}\text { Erosión de la } \\
\text { democracia }\end{array}$ \\
\hline Brasil & $1964-1985$ & & $1985-2010$ & & Democrático \\
\hline Chile & $1973-1990$ & & $1990-2010$ & & Democrático \\
\hline Colombia & $1949-1957$ & $\begin{array}{c}\text { Ola } \\
\text { democrática }\end{array}$ & & $1957-2010$ & $\begin{array}{l}\text { Estancamiento } \\
\text { de la democracia }\end{array}$ \\
\hline Ecuador & $1970-1978$ & $(1977-1994)$ & $1979-1999$ & $2000-2010$ & $\begin{array}{l}\text { Erosión de la } \\
\text { democracia }\end{array}$ \\
\hline E1 Salvador & $1900-1983$ & & $1994-2010$ & $1984-1993$ & Democrático \\
\hline Guatemala & $1954-1985$ & & & $1986-2010$ & $\begin{array}{l}\text { Estancamiento } \\
\text { de la democracia }\end{array}$ \\
\hline Honduras & $1963-1981$ & & $1999-2008$ & $1982-1998$ / $2009-2010$ & $\begin{array}{l}\text { Estancamiento } \\
\text { de la democracia }\end{array}$ \\
\hline México & $1913-1987$ & & $2000-2010$ & $1988-1999$ & Democrático \\
\hline Nicaragua & $1936-1983$ & & $1990-1996$ & $1984-1989$ / $1997-2010$ & $\begin{array}{l}\text { Erosión de la } \\
\text { democracia }\end{array}$ \\
\hline Panamá & $1968-1989$ & & $1994-2010$ & $1990-1993$ & Democrático \\
\hline Paraguay & $1900-1988$ & & $2008-2010$ & $1989-2007$ & Democrático \\
\hline $\begin{array}{l}\text { República } \\
\text { Dominicana }\end{array}$ & $1928-1977$ & & $1978-2010$ & & $\begin{array}{l}\text { Estancamiento } \\
\text { de la democracia }\end{array}$ \\
\hline Uruguay & $1973-1984$ & & $1985-2010$ & & Democrático \\
\hline Venezuela & $2009-2010$ & & & $1999-2008$ & $\begin{array}{l}\text { Erosión de la } \\
\text { democracia }\end{array}$ \\
\hline
\end{tabular}

* Algunos cambios han sido añadidos de acuerdo con lo ocurrido en los útimos seis años. En particular, la tendencia reeleccionista.

Fuente: Mainwaring y Pérez-Liñán (2013).

y por el otro, la flexibilización de la reelección presidencial. Durante los últimos 25 años varios países latinoamericanos cambiaron sus constituciones para favorecer el mecanismo de reelección presidencial ${ }^{6}$. Los últimos cambios parecen estar guiados por iniciativas personales que utilizan las reformas para satisfacer intereses políticos de corto plazo (Negretto, 2009; Gargarella, 2011). La popularidad de que gozan los mandatarios cuyo propósito es permanecer en el poder ha fortalecido esta tendencia reeleccionista en América Latina (Arenas y Valencia, 2009; Sánchez, 2013; 2015).

Ahora bien, la totalidad de las naciones latinoamericanas (exceptuando a México después de la revolución) han contemplado en su ordenamiento constitucional la figura de la reelección presidencial, y en su mayoría la han modificado entre dos y cuatro veces (ver cuadro 2). Hasta 1994, dos tendencias definidas pueden observarse: una inercial y otra restrictiva (Sánchez, 2013). Al interior de la primera, varios países mantuvieron la figura de la reelección presidencial alterna (con algunas variaciones) mientras otros prohibieron

a la reelección; por mencionar algunos casos: E1 Salvador (1931-1979), Brasil (1964-1985), Panamá (1968-1989), Chile (1973-1990) y Uruguay (1973-1985).

${ }^{6}$ Ver una discusión detallada sobre la reelección en América Latina en Sánchez (2013). 
Cuadro 2

Dinámica de la reelección presidencial en América Latina

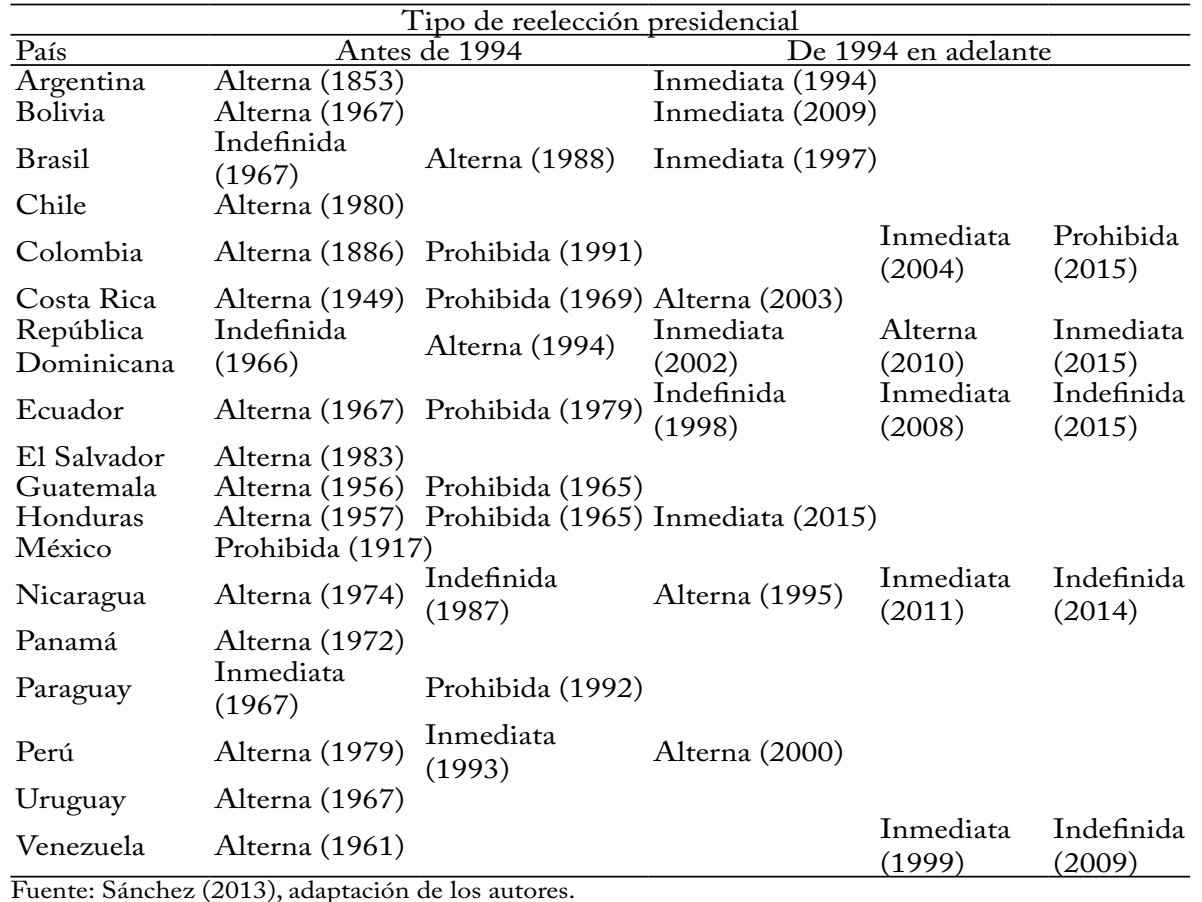

cualquier tipo de reelección presidencial. Para 1994, producto de la experiencia con los gobiernos presidenciales extensos, en muchos casos dictaduras de jure o de facto, la reelección presidencial inmediata había desaparecido en la región. Sin embargo, la tendencia desde entonces ha sido más permisiva con este mecanismo, con la característica adicional de que las nuevas disposiciones han sido avaladas por las altas cortes que se supone protegen los principios esenciales del acuerdo constitucional.

\section{LOS PRONUNCIAMIENTOS DE LAS ALTAS CORTES COMO MANIFESTACIÓN DE UN JUEZ MAXIMIZADOR DE RENTAS}

Actualmente catorce de dieciocho países latinoamericanos contemplan la figura de la reelección presidencial; seis de manera alterna, cinco de manera consecutiva (reelección inmediata) y tres de forma indefinida. La característica distintiva de los dos últimos grupos para alcanzar este objetivo ha sido la modificación constitucional a partir de 1994; usualmente por iniciativa de mandatarios que gozan de una amplia popularidad en sus países, y que cuentan con el apoyo o con- 
trol del juez constitucional. La influencia de personalidades políticas y la facilidad con la que se llevan a cabo las reformas en torno a la reelección dan origen a la provisionalidad institucional propia de los gobiernos latinoamericanos.

Perú fue pionero en esta materia y su caso es complejo. En 1993 se aprobó la reelección inmediata, al contemplar la posibilidad de que los expresidentes pudieran volver a ocupar el cargo una vez transcurrido un periodo después de su presidencia. Esta reforma, promovida por el presidente de entonces, Alberto Fujimori, fue reinterpretada por sus seguidores en el congreso en el sentido de que su segundo mandato (ganado en 1995) era el primero bajo el nuevo ordenamiento constitucional. Luego, cuando Fujimori aspiró a la presidencia por un tercer periodo consecutivo, en el año 2000, se consideró que eras su segundo periodo constitucional. Fujimori fue reelegido, pero se vio forzado a renunciar casi inmediatamente por los escándalos de corrupción y violación de derechos humanos. Ese mismo año, Perú volvió al sistema de reelección presidencial alterna.

Otros presidentes latinoamericanos siguieron el ejemplo peruano. El régimen constitucional en Argentina, que data de 1853, contemplaba la reelección presidencial alterna. No obstante, Carlos Menem, en 1994, promovió una reforma para permitir la reelección inmediata, sin excluir la posibilidad de un futuro periodo alterno. Menem fue reelegido. Por su parte, la constitución brasileña de 1988 (adoptada después del final de un gobierno militar) permitía solo la reelección alterna, pero en 1997, con el liderazgo de Fernando Enrique Cardoso, se aprobó la reelección presidencial inmediata por un solo periodo. Cardoso fue reelegido al año siguiente.

Por su parte, con la iniciativa de Hipólito Mejía, en el año 2002 República Dominicana aprobó la reelección presidencial inmediata por medio de una reforma que el Tribunal Constitucional juzgó conforme a la carta política. Honduras se unió al grupo reeleccionista y en abril de 2015 la sala constitucional de la Corte Suprema de Justicia aprobó la reelección. De igual manera, esta figura fue incorporada en la constitución promulgada en Bolivia por Evo Morales en 2009. En el caso boliviano, el Tribunal Constitucional Plurinacional aprobó un referendo que pretendía buscar el consentimiento de los bolivianos para la reelección de Evo Morales en las elecciones de 2019. A pesar de que Morales perdió el referendo, la pérdida de independencia de la rama judicial ha sido notoria (Vargas, 2015).

En Colombia, Álvaro Uribe Vélez fue reelegido en 2006 mediante una reforma constitucional aprobada por la Corte Constitucional; 
no obstante, en los últimos años revirtió la tendencia. Cuando los aliados políticos del expresidente Uribe promovieron una iniciativa popular para facilitar su segunda reelección, fue rechazada por la Corte Constitucional en 2010, con el argumento de la sustitución de la constitución. Juan Manuel Santos fue elegido como candidato muy cercano a Uribe y, sin embargo, después de su reelección en 2014, en 2015 propuso una reforma constitucional para prohibir la reelección presidencial.

El grupo de países que cuenta con reelección indefinida lo encabeza Venezuela. A petición del presidente Hugo Chávez, la regla de reelección alterna establecida en la constitución de 1961 fue modificada en 1999, y la reelección inmediata por dos periodos fue aprobada. Chávez fue reelegido en el año 2000 y en el año 2006. En 2009 fue propuesta la reelección indefinida, la cual se aprobó y Chávez fue reelegido una vez más en 2012. Al poco tiempo de iniciado su cuarto periodo, falleció. Por su parte, Ecuador aprobó la reelección presidencial inmediata en 2008 por iniciativa del entonces presidente Rafael Correa. Actualmente, con el aval de la Corte Constitucional, Ecuador contempla la reelección presidencial indefinida, pero regirá a partir de 2021. La reelección presidencial indefinida también fue aprobada en Nicaragua en 2014, después de que en 2011 Daniel Ortega fuera reelegido gracias a un pronunciamiento de la Corte Suprema que hacía caso omiso de la prohibición establecida en la constitución de dicho país.

Como puede observarse, los elementos transversales en los ejemplos citados son: la modificación de la constitución por iniciativa de personalidades que gozan de gran popularidad, y el débil papel que juega la rama judicial a la hora de velar por el cumplimiento de los periodos electorales y la estabilidad de la constitución (ver cuadro 3); circunstancia que puede explicarse por la capacidad que tiene el presidente de capturar a las otras ramas del poder público y así asegurar que sus iniciativas se lleven a cabo. El presidente es reelegido por el poder que concentra y no por su gestión, y además gana con amplios márgenes (Penfold, Corrales y Hernández, 2014); de diecinueve elecciones presidenciales que se llevaron a cabo en América Latina entre 1994 y 2014, donde uno de los candidatos era mandatario, en dieciocho casos la reelección tuvo lugar (Flórez, 2015). Esta realidad preocupa y desconcierta porque, como se mencionó, la huella que dejaron las dictaduras provocó un fuerte rechazo a las larga permanencia en el poder y una apuesta por la alternancia y la participación pluralista. 
Cuadro 3

Reelección presidencial en América Latina-Iniciativa y pronunciamiento del juez constitucional

\begin{tabular}{|c|c|c|c|}
\hline País & Reelección & Iniciativa & Pronunciamiento del juez \\
\hline Bolivia & Inmediata (2009) & Evo Morales & $\begin{array}{l}\text { Aprobatorio (Tribunal Constitu- } \\
\text { cional Plurinacional) }\end{array}$ \\
\hline Colombia & $\begin{array}{l}\text { Inmediata/Prohibida } \\
\quad(2004 / 2015)\end{array}$ & Alvaro Uribe & $\begin{array}{c}\text { Aprobatorio/restrictivo (Corte } \\
\text { Constitucional) }\end{array}$ \\
\hline Costa Rica & Alterna (2003) & Óscar Arias & $\begin{array}{l}\text { Aprobatorio (Sala Constitucional } \\
\text { Corte Suprema de Justicia) }\end{array}$ \\
\hline $\begin{array}{l}\text { República } \\
\text { Dominicana }\end{array}$ & Inmediata (2015) & $\begin{array}{l}\text { Leonel Fernández } \\
\text { Danilo Medina }\end{array}$ & Aprobatorio (Corte Constitucional) \\
\hline Ecuador & $\begin{array}{l}\text { Inmediata/Indefinida } \\
(2008 / 2015)\end{array}$ & Rafael Correa & Aprobatorio (Corte Constitucional) \\
\hline Honduras & Inmediata (2015) & $\begin{array}{l}\text { Juan Orlando } \\
\text { Hernandez }\end{array}$ & $\begin{array}{l}\text { Aprobatorio (Sala Constitucional } \\
\text { Corte Suprema de Justicia) }\end{array}$ \\
\hline Nicaragua & Indefinida (2014) & Daniel Ortega & $\begin{array}{c}\text { Aprobatorio (Corte Suprema de } \\
\text { Justicia) }\end{array}$ \\
\hline Venezuela & Indefinida (2009) & Hugo Chavez & $\begin{array}{c}\text { Aprobatorio (Tribunal Supremo de } \\
\text { Justicia) }\end{array}$ \\
\hline
\end{tabular}

Como sugiere el cuadro 3, en clara oposición a lo estipulado en las constituciones latinoamericanas, los pronunciamientos de la rama judicial han favorecido, en su mayoría, los intereses reeleccionistas de los presidentes. Muchas iniciativas para eliminar o debilitar los periodos electorales fueron ratificadas por los jueces constitucionales. Si un juez está dispuesto a aceptar la eliminación de estas disposiciones por iniciativa de las otras ramas del poder público, la independencia y la benevolencia del juez pueden ponerse en duda. Esta realidad conduce al análisis de la captura del juez por parte del ejecutivo, como muestra de la debilidad institucional en la cual la reelección resulta ser un mecanismo sumamente perjudicial para la democracia y el bienestar de los ciudadanos.

$\mathrm{E} 1$ caso venezolano puede ilustrar el argumento. Conforme el chavismo ha logrado controlar el Tribunal Supremo, los pronunciamientos de este órgano han favorecido los intereses del ejecutivo venezolano. Las denuncias acerca de la acción del tribunal en contra de la rama legislativa y la oposición en general, son muestra de la amenaza que pueden representar para la democracia la reelección presidencial y la acción de un juez constitucional empoderado pero sin independencia ${ }^{7}$.

Venezuela es el ejemplo más extremo y evidente; no obstante, como se observó, en el resto de países no resulta fácil distinguir la

7 Puede consultarse la prensa internacional que documenta al respecto, p. ej. El Universal (2013) y Semana (2017). 
naturaleza ética del juez al aprobar las reformas que dieron paso a la reelección presidencial. La experiencia histórica de América Latina sugiere que los periodos electorales límite fueron establecidos por razones legítimas de balance institucional. $\mathrm{Si}$, como ha sido frecuente, reelecciones sucesivas conducen a la concentración del poder en el presidente, y a un incremento en la capacidad del mandatario para influenciar otras ramas del poder, la ductilidad del juez constitucional es un factor determinante para la estabilidad de las instituciones. La presencia de un juez que funge como protector del acuerdo constitucional, aun cuando es un propósito loable, se erige sobre la noción de un mecanismo de supervisión ideal en cuanto a la naturaleza ética del juez se refiere. Este principio idealista conduce a sobrevalorar el rol que puede cumplir el juez como protector de la constitución. Una vez el supuesto de idoneidad del juez no se cumple, su poder y activismo conducen a un incremento del poder que concentra el presidente, y en consecuencia a un detrimento del bienestar de la población.

\section{CONSIDERACIONES FINALES}

Tres elementos analíticos han sido provistos para determinar si la reelección presidencial inmediata y el rol del juez constitucional funcionan como un mecanismo compuesto de rendición de cuentas en América Latina: la perspectiva teórica de principal-agente sobre el problema de rendición de cuentas; el examen histórico de la reelección presidencial y las largas duraciones en América Latina, y el análisis de los personalismos y los pronunciamientos del juez en la coyuntura reeleccionista.

Los resultados de la perspectiva teórica de agencia establecen que la eficacia de la reelección presidencial inmediata depende fundamentalmente de la valorización que hace el presidente de sus posibles rentas futuras, y de la naturaleza o tipo de juez, de la independencia que este último conserve frente al ejecutivo.

El componente histórico y el análisis coyuntural proveen evidencia para afirmar que el nivel de impaciencia u oportunismo del presidente es muy alto en América Latina. El fuerte presidencialismo; la influencia de los personalismos; las inclinaciones reeleccionistas; los gobiernos autoritarios; las dictaduras de jure o de facto, son evidencia de esa tendencia latinoamericana hacia la concentración del poder en el ejecutivo, dadas las rentas derivadas de la permanencia en el poder.

De igual modo, el establecimiento de un juez de corte benevolente no es realista, y por tanto los pronunciamientos del juez a favor de la 
reelección no pueden ser justificados como interpretaciones autónomas de la constitución. La imposibilidad de determinar si las acciones del juez constitucional buscan favorecer los intereses del presidente hace demasiado riesgoso construir un diseño institucional que confíe en el juez como mecanismo de refuerzo de la reelección para forzar a los políticos a rendir cuentas. La amenaza que representa para la institucionalidad latinoamericana un juez empoderado, sin independencia y maximizador de rentas, es evidente.

Estos resultados conducen a la conclusión de que la ciudadanía en América Latina no debe contemplar la reelección presidencial inmediata como mecanismo de rendición de cuentas, ni tampoco considerar el rol del juez constitucional como elemento complementario en el proceso de control político. Así, debe limitar el activismo del juez constitucional en lugar de exacerbarlo. Contrario a lo expuesto en algunos de los artículos citados sobre la importancia que han tenido los jueces constitucionales para fortalecer el imperio de la ley, este artículo considera que el ordenamiento jurídico de los países latinoamericanos no debe depender de la idoneidad ética del juez constitucional. La rama judicial ha de conservarse independiente, pero debe limitar su rol y no invadir las esferas de decisión de las demás ramas del poder público.

El fortalecimiento del proceso político de rendición de cuentas en América Latina no depende de la reelección presidencial ni del rol del juez constitucional; depende fundamentalmente de una relación consciente e informada entre la ciudadanía y sus representantes ${ }^{8}$. El panorama del control de los políticos resulta entonces poco alentador, en tanto los individuos pretenden perpetuarse en el poder, la rama legislativa no cumple su labor de control, el juez constitucional es proclive a la captura por parte del ejecutivo y los ciudadanos no tienen la información para exigir a sus representantes resultados.

$\mathrm{El}$ análisis propuesto en este artículo procura ilustrar un argumento sostenido por José Fernando Flórez Ruiz en su libro Todo lo que la democracia no es y lo poco que sí: defensa de una concepción democrática realista (2015). Es importante conocer la utilidad de los arreglos institucionales, pero sobre todo reconocer sus limitaciones. La idea-

8 En Mainwaring y Welna (2003) se encuentran discusiones sobre estos conceptos y su aplicación en América Latina, Moreno et al. (2003) muestran que la rendición de cuentas horizontal no existe. Subrayan la rendición de cuentas electoral y sostienen que la raíz del problema, y la causa de la sobrevaloración de las agencias de vigilancia, es la mala relación entre la ciudadanía y los políticos, porque si la rendición de cuentas vertical funcionara no habría necesidad de supervisores dentro del Estado. 
lización conduce a la decepción y a la percepción de inoperancia del Estado. La abstracción utilizada en la segunda sección buscaba establecer unas condiciones ideales que al contrastarlas con la realidad latinoamericana produjeran argumentos para refutar la utilidad de la reelección presidencial y precisar los alcances de un juez constitucional. El proceso político de rendición de cuentas en América Latina necesita de una concepción más realista sobre el carácter oportunista de los agentes que participan en el entorno político.

\section{REFERENCIAS BIBLIOGRÁFICAS}

Acemoglu, D., Robinson, J. y Johnson, S. (2005). Institutions as the fundamental cause of long-run economic growth. En P. Aghion y S. T. Durlauf (eds.), Handbook of economic growth (pp. 385-472). Amsterdam: North Holland.

Arenas, G. y Valencia, D. (2009). Elecciones y reelecciones presidenciales en América Latina. Perfil de Coyuntura Económica, 13, 76-96.

Baliga, S. (1999). Monitoring and collusion with "soft" information. The Journal of Law, Economics, and Organization, 15(2), 434-440.

Barro, R. (1973). The control of politicians: An economic model. Public Choice, 14, 19-42.

Baturo, A. (2014). Democracy, dictatorship, and term limits. Ann Arbor: The University of Michigan Press.

Berganza, J. C. (2000). Two roles for elections: Disciplining the incumbent and selecting a competent candidate. Public Choice, 105(1), 165-194.

Bernal, C. (2013). Unconstitutional constitutional amendments in the case study of Colombia: An analysis of the justification and meaning of the constitutional replacement doctrine. International Journal of Constitutional Law, 11(2), 339-357.

Bernal, C., Caicedo, A. y Serrafero, M. (2015). Reelección indefinida vs. democracia constitucional. Sobre los límites al poder de reforma constitucional en el Ecuador. Temas de Derecho Público, 95.

Besley, T. (2006). Principled agents? The political economy of good government. Oxford University Press on Demand.

Besley, T. y Case, A. (1995). Does electoral accountability affect economic policy choices? Evidence from gubernatorial term limits. The Quarterly Journal of Economics, 110(3), 769-798.

Brandt, U. S. y Svendsen, G. T. (2013). Why does bureaucratic corruption occur in the EU? Public Choice, 157(3-4), 585-599.

Carey, J. M. (2003). The reelection debate in Latin America. Latin American Politics and Society, 45(1), 119-133.

Carey, J. M. y Shugart, M. S. (eds.). (1998). Executive decree authority. Cambridge: Cambridge University Press.

Cooter, R. (2000). The strategic constitution. Princeton: Princeton University Press.

Cox, G. W. y Morgenstern, S. (2001). Latin America's reactive assemblies and proactive presidents. Comparative Politics, 33(2), 171-189. 
Dal Bó, E. (2006). Regulatory capture: A review. Oxford Review of Economic Policy, 22(2), 203-225.

Drazen, A. (2000). Political Economy in Macroeconomics. Princeton: Princeton University Press.

Drazen, A., Persson, T., Tabellini, G. et al. (2000). The "New political economy": Recent books by Allen Drazen and by Torsten Persson and Guido Tabellini. Journal of Economic Literature, 38(4), 915-925.

El Financiero. (2013, marzo 10). Reelección presidencial en Costa Rica cambió reglas de proceso político, [http://www.elfinancierocr.com/ economia-y-politica/hito-reeleccion_presidencial-Ruben_Hernandez_0_259174131.html].

El Nacional. (2015, marzo 15). Reforma constitucional y reelección presidencial, [http://elnacional.com.do/reforma-constitucional-yreeleccion-presidencial/].

El País. (2008, noviembre 30). Hugo Chávez reabre el proceso para su reelección indefinida, [https://elpais.com/internacional/2008/11/30/ actualidad/1227999611_850215.html].

El País. (2014, enero 28). Aprueban en Nicaragua reforma que permite reelección presidencial indefinida, [http://www.elpais.com.co/elpais/ internacional/noticias/aprueban-nicaragua-reforma-permite-reeleccion-indefinida].

El Tiempo (2010, febrero 26). La Corte Constitucional le dijo "no" al referendo reeleccionista: era Uribe terminará el 7 de agosto, [http:// www.eltiempo.com/archivo/documento/CMS-7304227].

El Tiempo (2014, octubre 31). Corte de Ecuador abre camino a reelección indefinida de Rafael Correa, http://www.eltiempo.com/mundo/ latinoamerica/corte-de-ecuador-abre-camino-a-reeleccion-indefinidade-correa/14773417].

El Universal (2013, enero 9). "Chavismo controla el Tribunal Supremo de Justicia”: exmagistrada, [http://www.eluniversal.com.co/cartagena/ internacional/chavismo-controla-el-tribunal-supremo-de-justiciaexmagistrada-104303].

Elster, J. (1994). The impact of constitutions on economic performance. Proceedings of the World Bank Annual Conference on Development Economics, pp. 209-226.

Emol (2015, octubre 28). Bolivia: Tribunal Constitucional aprueba plebiscito sobre reelección presidencial, http://www.emol.com/noticias/ Nacional/2015/10/29/756751/Bolivia-Tribunal-Constitucional-aprueba-plebiscito-que-extiende-periodo-presidencial.html].

Faure-G., A., Laffont. J. J. y Tirole, J. (2002). Risk averse supervisors and the efficiency of collusion. Contributions to Theoretical Economics, 2(1), 1-30.

Ferejohn, J. (1986). Incumbent performance and electoral control. Public Choice, 50, 5-25.

Ferraz, C. y Finan, F. (2011). Electoral accountability and corruption: Evidence from the audits of local governments. The American Economic Review, 101(4), 1274-1311. 
Flórez, J. F. (2015). Todo lo que la democracia no es y lo poco que sí: defensa de una concepción democrática realista. Bogotá: Universidad Externado de Colombia.

Gailmard, S. (2012). Accountability and principal-agent models. En M. Bovens, R. Goodin y T. Schillemans (eds.). The Oxford Handbook of Public Accountability. Oxford: Oxford Universtity Press.

Gargarella, R. (2011). El constitucionalismo latinoamericano y la "sala de máquinas” de la constitución (1980-2010). Gaceta Constitucional, 48, 289-306.

Gargarella, R. y Courtis, C. (2009). El nuevo constitucionalismo latinoamericano: promesas e interrogantes. Santiago de Chile: Naciones Unidas.

Ginsburg, T., Melton, J. y Elkins, Z. (2010). On the evasion of executive term limits. Public and Legal Theory Working Papers, 328, 1-45.

González, J. (2008). Hurwicz y el juez de última instancia. Revista de Economia Institucional, 10(19), 115-129.

Henao, J. C. (2013). El juez constitucional: un actor de las políticas públicas. Revista de Economía Institucional, 15(29), 67-102.

Hurwicz, L. (2008). But who will guard the guardians. The American Economic Review, 98(3), 577-585.

Kapiszewski, D. y Taylor, M. M. (2008). Doing courts justice? Studying judicial politics in Latin America. Perspectives on Politics, 6(4), 741-767.

Kenney, C. D. (2003). Horizontal accountability: Concepts and conflicts. En S. Mainwaring y C. Welna (eds.). Democratic accountability in Latin America. Nueva York: Oxford University Press.

Laffont, J. J. (1999). Political economy, information and incentives. European Economic Review, 43(4), 649-669.

Laffont, J. J. (2000). Incentives and political economy. Nueva York: Oxford University Press.

Laffont, J. J. y Martimort, D. (1998). Transaction costs, institutional design and the separation of powers. European Economic Review, 42(3), 673-684.

Laffont, J. J. y Martimort, D. (1999). Separation of regulators against collusive behavior. The Rand Journal of Economics, 3O(2), 232-262.

Laffont, J. J. y Martimort, D. (2006). The theory of incentives. The principalagent model. Princeton: Princeton University Press.

Laffont, J. J. y Meleu, M. (2001). Separation of powers and development. Journal of Development Economics, 64(1), 129-145.

Laffont, J. J. y Tirole, J. (1990). The politics of government decision making: Regulatory institutions. Journal of Law, Economics, and Organization, 6(1), 1-31.

Laffont, J. J. y Tirole, J. (1991). The politics of government decisionmaking: A theory of regulatory capture. The Quarterly Journal of Economics, 106(4), 1089-1127.

Laffont, J. J. y Tirole, J. (1993). A theory of incentives in procurement and regulation. Cambridge, Mass. Londres: MIT Press.

Landau, D. (2010). Political institutions and judicial role in comparative constitutional law. Harvard International Law Journal, 51(2), 319-377.

Madison, J. (1787-1788). The Federalist, 10. November, 22. 
Magaloni, B. (2003). Authoritarianism, democracy and the supreme court: Horizontal exchange. En S. Mainwaring y C. Welna (eds.). Democratic accountability in Latin America, NuevaYork: Oxford University Press.

Mainwaring, S. y Pérez-L., A. (2013). Democracies and dictatorships in Latin America: Emergence, survival, and fall. Nueva York: Cambridge University Press.

Mainwaring, S. y Welna, C. (eds.). (2003). Democratic accountability in Latin America. Nueva York: Oxford University Press.

Martínez-B., E. (2009). Seeking the political role of the third government branch: A comparative approach to high courts in Central America. Saarbrücken: VDM Verlag.

Martínez-B., E. (2010). Las cortes supremas como mecanismo de distribución de poder: el caso de la reelección presidencial en Costa Rica y Nicaragua. Revista de Ciencia Politica, 30(3), 723-750.

Martínez-B., E. y A. Brenes (2012), "Y volver, volver, volver...”. Un análisis de los casos de intervención de las cortes supremas en la reelección presidencial en Centroamérica. Anuario de Estudios Centroamericanos, 38, 109-136.

Maskin, E. y Tirole, J. (2004). The politician and the judge: Accountability in government. The American Economic Review, 94(4), 1034-1054.

Moreno, E., Crisp, B. F. y Shugart, M. S. (2003). The accountability deficit. En S. Mainwaring y C. Welna (eds.), (2003). Democratic accountability in Latin America, Nueva York: Oxford University Press.

Negretto, G. (2009). Paradojas de la reforma constitucional en América Latina. Journal of Democracy en Español, 1(1), 38-54.

Nunes, R. M. (2010). Ideational origins of progressive judicial activism: The Colombian Constitutional Court and the right to health. Latin American Politics and Society, 52(3), 67-97.

O'Donnell, G. (2003). Horizontal accountability: The legal institutionalization of mistrust. En S. Mainwaring y C. Welna (eds.), Democratic accountability in Latin America. Oxford: Oxford University Press.

Penfold, M., Corrales, J. y Hernández, G. (2014). Los Invencibles: la reelección presidencial y los cambios constitucionales en América Latina. Revista de Ciencia Política, 34(3), 537-559.

Pérez, M. (2007). Una aproximación a la teoría económica del derecho constitucional. Revista Derecho del Estado, 20(3).

Persson, T. y Tabellini, G. (2000). Political economics: Explaining economic policy. Cambridge: MIT Press.

Przeworski, A., Stokes, S. C. y Manin, B. (eds.). (1999). Democracy, accountability, and representation, vol. 2. Nueva York: Cambridge University Press.

Roznai, Y. (2017). Unconstitutional constitutional amendments: The limits of amendment powers. Nueva York: Oxford University Press.

Samuels, D. y Hellwig, T. (2010). Elections and accountability for the economy: A conceptual and empirical reassessment. Journal of Elections, Public Opinion and Parties, 20(4), 393-419.

Sánchez, I. T. (2013). Las reformas a la reelección presidencial en América Latina. Estudios Sociológicos, 31(91), 59-85. 
Sánchez, I. T. (2015). Las reformas a la reelección presidencial del nuevo siglo en América Central: tres intentos de reforma y un golpe de Estado. Politica y Gobierno, 22(1), 147-173.

Schor, M. (2009). An essay on emergence of constitutional courts: The cases of Mexico and Colombia. Indiana Journal of Global Legal Studies, 16(1), 1-22.

Semana (2017, mayo 18). Estos son los jefes judiciales de Venezuela a los que EE.UU. congeló los bienes, [http://www.semana.com/ mundo/articulo/estados-unidos-congela-bienes-de-magistradosvenezolanos/525671].

Serrafero, M. (2011). La reelección presidencial indefinida en América Latina. Revista de Instituciones, Ideas y Mercados, 54, 225-259.

Shapiro, M. y Sweet, A. S. (2002). On law, politics, and judicialization. Oxford, NuevaYork: Oxford University Press.

Smart, M. y Sturm, D. (2013). Term limits and electoral accountability. Journal of Public Economics, 107, 93-102.

Stigler, G. J. (1971). The theory of economic regulation. The Bell Journal of Economics and Management Science, 2(1), 3-21.

Strausz, R. (1997). Collusion and renegotiation in a principal-supervisoragent relationship. The Scandinavian Journal of Economic, 4, 497-518.

Streb, J. (1999). Reelection or term limits? The short and the long view of economic policy. CEMA Working Papers, Serie Documentos de Trabajo, 144, 1-28.

Streb, J. (2005). Signaling in political budget cycles: How far are you willing to go? Journal of Public Economic Theory, 7(2), 229-252.

Tirole, J. (1986). Hierarchies and bureaucracies: On the role of collusion in organizations. The Journal of Law, Economics and Organization, 2(2), 181-214.

Uprimny, R. (2007). La judicialización de la política en Colombia: casos, potencialidades y riesgos. Justicia, politica y derechos en América Latina.

Vargas L. A. E. (2015). La reelección presidencial en la jurisprudencia del Tribunal Constitucional Plurinacional de Bolivia. La ilegítima mutación de la constitución a través de una ley de aplicación normativa. Iuris Tantum. Revista Boliviana de Derecho, 19, 446-469.

Zielinski, J., Slomczynski, K. y Shabad, G. (2005). Electoral control in new democracies: The perverse incentives of fluid party systems. World Politics, 57(3), 365-395. 